\title{
Enhanced therapeutic effect of transcatheter arterial chemoembolization combined with radioactive I-125 seed implantation on liver cancer
}

\author{
WENBAO LIU, NANGUANG XIU, JINWEI ZHAO and LIANHAO ZHAO \\ Department of Intervention Therapy, Laiyang Central Hospital, Yantai, Shandong 265200, P.R. China
}

Received December 3, 2018; Accepted January 30, 2020

DOI: $10.3892 / \mathrm{ol} .2020 .11765$

\begin{abstract}
The present study aimed to evaluate the therapeutic effect of transcatheter arterial chemoembolization (TACE) combined with radioactive I-125 (iodine-125) seed implantation on liver cancer. A total of 38 liver cancer patients in the combined treatment group were treated with lobaplatin-TACE combined with radioactive I-125 seed implantation, while 45 patients in the TACE group were treated only with lobaplatin-TACE. Patients were followed up for survival time, and the liver function, change in $\alpha$-fetoprotein (AFP) and side effects were evaluated. The average survival time of patients was 6.1 months in the TACE group and 8.2 months in the combined treatment group, and the overall survival was significantly different between the two groups $(\mathrm{P}<0.05)$. The response rate of lesions in the combined treatment group was superior to that observed in the TACE group, and the change in AFP in the TACE group was smaller than that in the combined treatment group. Improvement in the effects on most liver function indices in the combined treatment group were better than those in the TACE group. In addition, there were no differences in the side effects experienced between the two groups. Results of this study indicate that the effect of TACE combined with radioactive $\mathrm{I}-125$ seed implantation is superior to that of TACE alone for the treatment of liver cancer, which can further benefit patients.
\end{abstract}

\section{Introduction}

Liver cancer is the sixth most common cancer in the world, and is the third leading cause of cancer-related mortality $(1,2)$. The incidence rate of complications due to liver cancer is extremely high. Currently, the various therapeutic regimens for liver cancer, include surgical treatment (liver resection and liver transplantation) and local treatment [transcatheter arterial chemoembolization (TACE) and ablation therapy] (3-5). When

Correspondence to: Dr Lianhao Zhao, Department of Intervention Therapy, Laiyang Central Hospital, 111 Changshan Road, Yantai, Shandong 265200, P.R. China

E-mail: cuan063@126.com

Key words: liver cancer, transcatheter arterial chemoembolization, radioactive I-125 seed implantation the tumor is in the late stage, surgical resection is often limited due to poor liver function and tumor invasion into blood vessels and bile duct. The curative effect of TACE on liver cancer has been widely accepted, and TACE can effectively control the development of cancer and improve the quality of life of these patients $(6,7)$. However, TACE must be performed repeatedly and causes unbearable liver damage for most patients. Traditional cytotoxic drugs are characterized by great toxicity and side effects and poor repeatability. Lobaplatin used in the treatment of liver cancer is the third-generation platinum with less adverse side effects (8). Iodine-125 (I-125) is mainly characterized by constant low-energy X-ray and $\gamma$-ray emissions, which inhibits or kills nearby tumor cells within a short distance and causes little damage to surrounding tissues. A higher local control rate and a lower incidence rate of complications can be obtained in radioactive I-125 seed implantation in the treatment of malignant tumors. In addition, it can improve the quality of life and prolong the survival time of patients (9). Radioactive I-125 (iodine-125) seed implantation has been used as a standard therapeutic method for early prostate cancer in European and American countries, and it is even used in the treatment of systemic solid tumors and has been found to have a therapeutic effect on liver cancer in China (10-13).

This study aimed to evaluate the combined treatment of TACE and radioactive I-125 seed implantation for the treatment of liver cancer and evaluate the therapeutic effect.

\section{Patients and methods}

Patient data. A total of 83 patients with liver cancer were treated with lobaplatin-TACE and/or I-125 seed implantation from January 2010 to February 2012. All procedures in this study were approved by the Ethics Committee of Laiyang Central Hospital. Histological type of all the selected patients was hepatocellular carcinoma. Patients were divided into a TACE group (only treated with lobaplatin-TACE, $n=45$ ) and a TACE + I-125 group (treated with lobaplatin-TACE combined with I-125 seed implantation, $n=38$ ). Age, gender, tumor size and number in both groups are documented in Table I.

Inclusion criteria included: i) Patients aged $\leq 75$ years, ii) patients diagnosed with primary liver cancer via imaging examination, tumor markers and/or histological examination, iii) patients who could not tolerate surgery due to physical conditions or the high difficulty of surgery (there existed more 
Table I. Characteristics of the liver cancer patients enrolled in the present study.

\begin{tabular}{|c|c|c|c|c|}
\hline Characteristics & TACE group & TACE + I-125 group & $\chi^{2}$ & P-value \\
\hline Age (years) & 45 & 38 & 0.000 & 0.991 \\
\hline$>50$ & 26 & 22 & & \\
\hline$\leq 50$ & 19 & 16 & & \\
\hline Sex & & & 0.002 & 0.961 \\
\hline Male & 27 & 23 & & \\
\hline Female & 18 & 15 & & \\
\hline Tumor diameter $(\mathrm{cm})$ & & & 0.007 & 0.933 \\
\hline$<5$ & 15 & 13 & & \\
\hline$\geq 5$ & 30 & 25 & & \\
\hline Number of tumor nodules & & & 0.548 & 0.459 \\
\hline 1 & 10 & 6 & & \\
\hline$>1$ & 35 & 32 & & \\
\hline Tumor capsule & & & 0.010 & 0.922 \\
\hline Complete & 24 & 19 & & \\
\hline Incomplete & 23 & 19 & & \\
\hline Classification of liver function & & & 0.096 & 0.757 \\
\hline A & 7 & 5 & & \\
\hline B & 38 & 33 & & \\
\hline
\end{tabular}

TACE group, patients treated with lobaplatin-TACE alone. TACE + I-125 group, patients treated with lobaplatin-TACE combined with radioactive I-125 (iodine-125) seed implantation. TACE, transcatheter arterial chemoembolization.

Table II. Comparison of the response rate of foci between the TACE and TACE + I-125 group of liver cancer patients at 1 month after surgery.

\begin{tabular}{lcccr}
\hline Response rate & TACE group & TACE + I-125 group & $\chi^{2}$ & P-value \\
\hline Number of foci & 145 & 142 & & \\
CR, $\mathrm{n}(\%)$ & $22(20.0)$ & $36(25.4)$ & 4.7 & 0.030 \\
PR, n (\%) & $41(28.3)$ & $58(40.8)$ & 5.0 & 0.025 \\
SD, n (\%) & $45(26.2)$ & $29(20.4)$ & 3.4 & 0.065 \\
PD, $\mathrm{n}(\%)$ & $37(25.5)$ & $17(11.7)$ & 8.6 & 0.003 \\
\hline
\end{tabular}

TACE group, patients treated with lobaplatin-TACE alone. TACE + I-125 group, patients treated with lobaplatin-TACE combined with radioactive I-125 (iodine-125) seed implantation. TACE, transcatheter arterial chemoembolization; CR, complete response; PR, partial response; $\mathrm{SD}$, stable disease; $\mathrm{PD}$, progressive disease.

than 3 tumor foci or the remaining liver could not maintain normal function after surgery), iv) patients with Child-Pugh classification of liver function in grade $\mathrm{A} / \mathrm{B}, \mathrm{v}$ ) patients with normal bone marrow function, vi) patients with expected survival time of not $<3$ months, vii) patients who signed the informed consent, and viii) patients with complete case data.

Exclusion criteria included: i) Patients who also underwent other interventional therapies, ii) patients who could not receive treatment due to severe complications, iii) patients who dropped out of the study.

TACE procedure. The Seldinger technique was used for TACE. In brief, the catheter was inserted via the femoral artery to the appropriate hepatic artery or superior mesenteric artery.
Angiography was performed to determine the blood supply of the tumor. The iodized oil emulsifier containing lobaplatin (5-10 $\mathrm{ml}$ iodized oil and $50 \mathrm{ml}$ lobaplatin fully mixed to form emulsifier) was injected (20-50 mg/time, $33.3 \mathrm{mg}$ on average) into the supplying artery of the tumor through the catheter once a month for the entire 12 month follow-up. The embolic agents included sodium alginate microspheres and iodized oil. Patients in the TACE group were treated with the same TACE procedure alone as that in the combined treatment group.

Radioactive I-125 seed implantation. I-125 seeds were generally implanted into patients at 2-4 weeks after TACE. Conventional computed tomography (CT) scan was performed to determine the site of the I-125 seed implantation, and the seed 
Table III. Survival time and survival rate of the liver patients in the TACE and TACE+I-125 group.

\begin{tabular}{|c|c|c|c|c|c|c|c|}
\hline \multirow[b]{2}{*}{ Group } & \multicolumn{4}{|c|}{ Survival rate $(\%)$} & \multirow{2}{*}{$\begin{array}{l}\text { Average survival } \\
\text { time (months) }\end{array}$} & \multirow[b]{2}{*}{$\chi^{2}$} & \multirow[b]{2}{*}{ P-value } \\
\hline & 3-month & 6-month & 9-month & 12-month & & & \\
\hline TACE & 100 & 36.4 & 9.1 & 9.1 & 6.1 & 4.0 & 0.04 \\
\hline TACE + I-25I & 100 & 72.7 & 27.3 & 18.2 & 8.2 & & \\
\hline
\end{tabular}

TACE group, patients treated with lobaplatin-TACE alone. TACE + I-125 group, patients treated with lobaplatin-TACE combined with radioactive I-125 (iodine-125) seed implantation. TACE, transcatheter arterial chemoembolization.

A

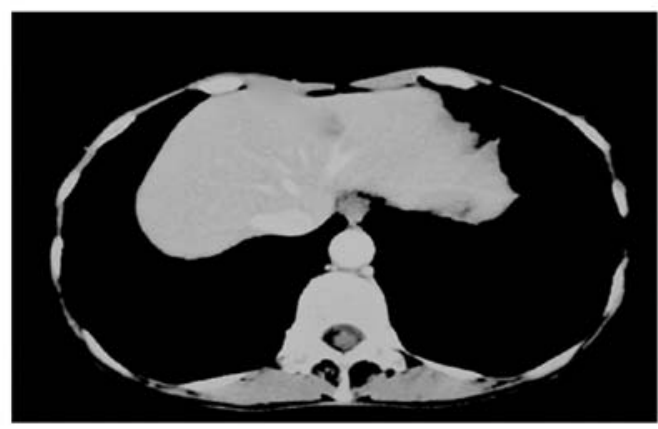

B



Figure 1. Computed tomography (CT) image of a patient. (A) Foci in a patient. (B) Foci in a patient at 1 month after surgery.

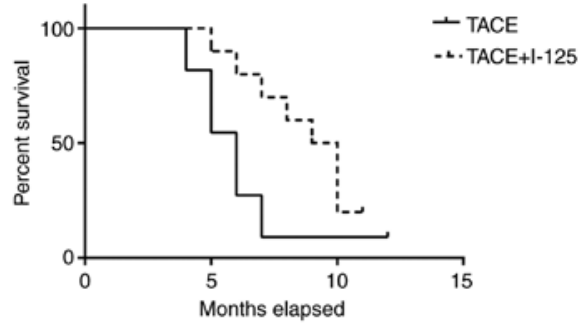

Figure 2. Survival curves of the liver cancer patients in the TACE and TACE + I-125 groups. TACE group, patients treated with lobaplatin-TACE alone. TACE + I-125 group, patients treated with lobaplatin-TACE combined with radioactive I-125 (iodine-125) seed implantation. TACE, transcatheter arterial chemoembolization.

was implanted as follows. According to the size, shape and site of the tumor, the appropriate puncture point was determined, and the treatment plan was developed. The needle implanted in the seed was inserted into the tumor tissue under the guidance of CT based on the number of tumors, radiation dose and distribution of the I-125 seed. The average energy of the seeds was $35.5 \mathrm{KeV}$, the half-life was 56 days, the radioactivity was $0.7 \mathrm{mCi}$, the tissue penetration ability was $1.7 \mathrm{~cm}$, and the dosage range of HCC particles was 140-160 Gy. When the tip of the needle reached the predetermined target zone and it was approximately $0.5 \mathrm{~cm}$ away from the distal end, the seeds were implanted backwards at an interval of $0.5-1.0 \mathrm{~cm}$.

Evaluation criteria. The effect of surgery was evaluated according to the Response Evaluation Criteria in Solid Tumors (RECIST) (14): i) Complete response (CR), ii) partial response $(\mathrm{PR})$, iii) stable disease (SD), and iv) progressive disease (PD).
Follow-up. At 3-7 days after treatment and before discharge, the biochemical indices of liver function and clinical manifestations (ascites, jaundice) were observed. Blood examination was performed once every 4 weeks after discharge, and the patients were followed up for not $<12$ months. One patient in the control group withdrew from the study, thus 44 patients in the control group were followed up.

Statistical analysis. GraphPad Prism statistical software 7.0 (GraphPad Software, Inc.) was used for analysis of all data. The measurement data are expressed as mean \pm SD and the t-test was used. Dunnett test was used to compare AFP expression among the groups. Pearson Chi-square test was adopted to compare general data and postoperative complications. The overall survival rate was calculated in both groups, and the difference was detected via log-rank test. $\mathrm{P}<0.05$ indicated that the difference was statistically significant.

\section{Results}

Therapeutic effect. The lesions in the two groups were evaluated one month after I-125 seed implantation in the TACE + I-125 group. In the TACE group, there were a total of 145 foci in the liver in 44 patients, including 22 cases of CR, 41 cases of PR, 45 cases of SD and 37 cases of PD. In the TACE + I-125 group, there were a total of 142 foci in the liver in 38 patients, including 36 cases of CR, 58 cases of PR, 29 cases of SD and 17 cases of PD. There were statistically significant differences between the two groups $(\mathrm{P}<0.05)$. (Table II). Fig. 1 shows the lesions of one patient in the TACE + I-125 group before and after one month of I-125 seed implantation. 
Table IV. Liver function indices in the TACE and TACE + I-125 group of liver cancer patients.

\begin{tabular}{llcccc}
\hline Group & \multicolumn{1}{c}{ Time } & ALT (U/l) & AST (U/l) & ALB (g/l) & TBIL $(\mu \mathrm{mol} / \mathrm{l})$ \\
\hline TACE & Before surgery & $112 \pm 34$ & $102 \pm 43$ & $20 \pm 16$ & $95 \pm 37$ \\
& 1 month after surgery & $87 \pm 43$ & $65 \pm 26$ & $22 \pm 19$ & $36 \pm 24$ \\
TACE + I-125 & Before surgery & $109 \pm 49^{\mathrm{b}}$ & $105 \pm 37^{\mathrm{b}}$ & $21 \pm 13^{\mathrm{b}}$ & $97 \pm 45^{\mathrm{b}}$ \\
& 1 month after surgery & $63 \pm 38^{\mathrm{a}}$ & $51 \pm 33^{\mathrm{a}}$ & $38 \pm 27^{\mathrm{a}}$ & $33 \pm 19$ \\
\hline
\end{tabular}

${ }^{a} \mathrm{P}<0.05$, significant difference compared with the TACE group 1 month after surgery. ${ }^{\mathrm{b}} \mathrm{P}>0.05$, no significant difference compared with the TACE group before surgery. ALT, alanine aminotransferase; AST, aspartate aminotransferase; ALB, albumin; TBIL, total bilirubin. TACE group, patients treated with lobaplatin-TACE alone. TACE + I-125 group, patients treated with lobaplatin-TACE combined with radioactive I-125 (iodine-125) seed implantation. TACE, transcatheter arterial chemoembolization.

Table V. AFP expression level (in $\mu \mathrm{g} / \mathrm{l}$ ) at different time points in in the TACE and TACE + I-125 groups of the liver cancer patients.

\begin{tabular}{lccccc}
\hline Group & 0 month & 3 months & 6 months & 9 months & 12 months \\
\hline TACE & $1,457 \pm 563$ & $765 \pm 262$ & $342 \pm 193$ & $165 \pm 54$ & $79 \pm 34$ \\
TACE + I-125 & $1,263 \pm 498$ & $511 \pm 143$ & $143 \pm 87$ & $73 \pm 49$ & $36 \pm 29$ \\
t & 2.23 & 5.74 & 9.54 & 8.45 & 10.67 \\
P-value & 0.678 & 0.034 & $<0.001$ & $<0.001$ & $<0.001$ \\
\hline
\end{tabular}

AFP, $\alpha$-fetoprotein. TACE group, patients treated with lobaplatin-TACE alone. TACE + I-125 group, patients treated with lobaplatin-TACE combined with radioactive I-125 (iodine-125) seed implantation. TACE, transcatheter arterial chemoembolization.

Table VI. Occurrence of side effects in the TACE and TACE + I-125 group of liver cancer patients.

\begin{tabular}{lcccccr}
\hline Group & $\mathrm{n}$ & $\begin{array}{c}\text { Bone marrow } \\
\text { suppression }\end{array}$ & $\begin{array}{c}\text { Nausea and } \\
\text { vomiting }\end{array}$ & $\begin{array}{c}\text { Liver } \\
\text { dysfunction }\end{array}$ & Diarrhea & P-value \\
\hline TACE & 44 & 28 & 39 & 44 & 31 & 0.45 \\
TACE + I-125 & 38 & 26 & 34 & 38 & 29 & \\
\hline
\end{tabular}

TACE group, patients treated with lobaplatin-TACE alone. TACE + I-125 group, patients treated with lobaplatin-TACE combined with radioactive I-125 (iodine-125) seed implantation. TACE, transcatheter arterial chemoembolization.

Survival time and survival rate. At 3,6,9 and 12 months, the survival rates in the TACE group were 100.0, 36.4, 9.1 and $9.1 \%$, respectively, and the survival rates were 100.0, 72.7, 27.3 and $18.2 \%$, respectively, in the TACE+I-125 group (Table III). The average survival time was 6.1 months in the TACE group and 8.2 months in the TACE+I-125 group. Both groups were followed up for 12 months. There were statistically significant differences between the two groups $(\mathrm{P}<0.05)$ (Fig. 2).

Liver function after surgery. The liver function indices for alanine aminotransferase, aspartate aminotransferase (AST), albumin (ALB) and total bilirubin (TBIL) were analyzed and compared at 1 month after surgery between the two groups (Table IV). Improvements in most liver function indices in the TACE + I-125 group were superior to those in the TACE group, displaying significant differences $(\mathrm{P}<0.05)$.
Changes in $\alpha$-fetoprotein $(A F P)$. As shown in Table $\mathrm{V}$, the AFP levels in the TACE and TACE + I-125 groups were $1,457 \pm 563$ and $1,263 \pm 498 \mu \mathrm{g} / 1$, respectively, before treatment, with no significant difference $(\mathrm{P}>0.05)$. After three months of the different treatments, the AFP in the TACE and TACE + I-125group decreased to $765 \pm 262$ and $511 \pm 143 \mu \mathrm{g} / 1$, respectively, with significant difference $(\mathrm{P}<0.05)$. At 6 and 9 months, there was a continuous decrease in both groups, but more significant in the TACE + I-125 group, and the difference between the two groups was significant $(\mathrm{P}<0.05)$. At 12 months, the AFP was $79 \pm 34 \mu \mathrm{g} / \mathrm{l}$ in the TACE group, and $36 \pm 29 \mu \mathrm{g} / \mathrm{l}$ in the TACE + I-125 group, with significant differences between the two groups $(\mathrm{P}<0.05)$.

Postoperative side effects in both groups. Side effects were present to a certain degree in both groups of patients (Table VI), but they were all alleviated after treatment. The occurrence of 
side effects had no significant difference between the TACE and TACE + I-125 group.

\section{Discussion}

Transcatheter arterial chemoembolization (TACE) is considered as one of the major treatment means of advanced liver cancer. However, drugs used in TACE are usually characterized by high toxicity and non-significant curative effect. As the third-generation platinum drug, lobaplatin also possesses a broad-spectrum antitumor effect, which has been used as a first-line chemotherapeutic agent in the treatment of a variety of tumors. In the present study, it was found that lobaplatin could be mixed with iodipin to become a more stable emulsifier, thus iodipin could be deposited more completely, obtaining a better curative effect.

Radioactive seed implantation is characterized by small trauma, a high therapeutic dose provided in the tumor target zone and only little damage to normal tissues. In recent years, radioactive seed implantation has been applied more widely in the treatment of malignant solid tumors, obtaining a superior therapeutic effect $(15,16)$. The development of I-125 seed implantation from the initial application in the treatment of early prostate cancer to the application in the treatment of systemic solid tumors is closely related to its antitumor effect. The principle is that the seeds carrying I-125 are directly implanted into the focus for continuous low-dose radiation on tumor cells, thus killing tumor cells through free oxygen radicals and ionization (17). The I-125 seed implantation can arrest the cell cycle in the radiation-sensitive $\mathrm{G} 2 / \mathrm{M}$ phase, thus killing tumor cells (18). In addition, the repeated seed implantation may have a 'superimposed effect', leading to the sharp increase in the radiation dose in the focus, thus inactivating tumor cells more completely. More importantly, the effective radiation radius of $\mathrm{I}-125$ is only $17 \mathrm{~mm}$, thus a higher cumulative dose in the tumor can be maintained, and I-125 can better act on tumor cells almost without influence on normal tissues and adjacent organs around the focus $(19,20)$. In addition, the I-125 implantation activates the cluster of differentiation $(\mathrm{CD}) 3^{+}$and $\mathrm{CD}^{+}$cells, thus producing an antitumor immune response. It was found in a randomized controlled study that I-125 brachytherapy after liver resection obviously prolongs the recurrence time and increases the overall survival of patients (21). Nag et al observed a high complete response (CR) or partial response (PR) rate for unresectable hepatocellular carcinoma in I-125 brachytherapy (22).

The advantage of TACE combined with I-125 seed implantation lies in that advantages of both therapeutic methods can be combined to further exert an antitumor effect. I-125 seed implantation can further inactivate residual tumor cells after TACE. I-125 seed implantation is necessary to be combined for tumors with insufficient blood supply, especially for patients who cannot undergo another TACE due to different structures of the hepatic artery and/or new vessels or patients with unsatisfactory iodipin deposition, so as to obtain superior effects. In the present study, PR rate in the TACE + I-125 group was higher than that in the TACE group, and the average survival time in the TACE + I-125 group was prolonged by 2.1 months compared with that in the TACE group, displaying statistically significant differences $(\mathrm{P}<0.05)$.
TACE combined with I-125 seed implantation reduces the frequency of the use of TACE, thereby reducing TACE-induced liver dysfunction, consequently having a positive impact on the long-term survival of patients. After I-125 seed implantation, the increased level of aspartate transaminase was lower, and the possible reason is the puncture needle-induced injury during implantation. The continuous radiation of I-125 seeds also leads to liver injury, but severe liver injury or other severe complications caused by I-125 seed implantation have not been reported (23-26).

Some studies have demonstrated that TACE combined with I-125 seed implantation can control not only the intrahepatic lesion but also the extrahepatic metastasis in patients with distant metastasis. Different treatment methods may lead to different final survival outcome, but I-125 seed implantation can completely inactivate the metastatic solid tumor, and reduce the burden of tumor on the whole body, which is of positive significance in the quality of life and survival time of patients.

In conclusion, TACE combined with I-125 seed implantation can better control the lesion and improve the survival rate of patients in the treatment of liver cancer compared with TACE alone, thus it is an effective combination treatment method. To better perform research on this technique, a prospective controlled study with a larger sample size and longer follow-up time is needed to verify the treatment effect.

\section{Acknowledgements}

Not applicable.

\section{Funding}

No funding was received.

\section{Availability of data and material}

The datasets used and/or analyzed during the current study are available from the corresponding author on reasonable request.

\section{Authors' contributions}

WL ad NX collected the general data of the patients and performed the treatment procedures. JZ evaluated the effects of the procedures in the two patient groups. WL and LZ conducted and analyzed data of the patient follow-up. All authors read and approved the final manuscript. All authors read and approved the manuscript and agree to be accountable for all aspects of the research in ensuring that the accuracy or integrity of any part of the work are appropriately investigated and resolved.

\section{Ethics approval and consent to participate}

The study was approved by the Ethics Committee of Laiyang Central Hospital Yantai, Shandong, China) and informed consents were signed by all patients and/or their guardians.

\section{Patient consent for publication}

The patients have provided consent for publication of images. 


\section{Competing interests}

The authors declare that they have no competing interests.

\section{References}

1. Parkin DM, Bray F, Ferlay J and Pisani P: Global cancer statistics, 2002. CA Cancer J Clin 55: 74-108, 2005.

2. Tang ZY, Ye SL, Liu YK, Qin LX, Sun HC, Ye QH, Wang L, Zhou J, Qiu SJ, Li Y, et al: A decade's studies on metastasis of hepatocellular carcinoma. J Cancer Res Clin Oncol 130: 187-196, 2004.

3. Bruix J and Sherman M; Practice Guidelines Committee, American Association for the Study of Liver Diseases: Management of hepatocellular carcinoma. Hepatology 42: 1208-1236, 2005.

4. Forner A, Llovet JM and Bruix J: Hepatocellular carcinoma. Lancet 379: 1245-1255, 2012.

5. Shi M, Lu LG, Fang WQ, Guo RP, Chen MS, Li Y, Luo J, $\mathrm{Xu}$ L, Zou RH, Lin XJ and Zhang YQ: Roles played by chemolipiodolization and embolization in chemoembolization for hepatocellular carcinoma: Single-blind, randomized trial. J Natl Cancer Inst 105: 59-68, 2013

6. Llovet JM and Bruix J: Systematic review of randomized trials for unresectable hepatocellular carcinoma: Chemoembolization improves survival. Hepatology 37: 429-442, 2003.

7. Raoul JL, Forner A, Bolondi L, Cheung TT, Kloeckner R and de Baere T: Updated use of TACE for hepatocellular carcinoma treatment: How and when to use it based on clinical evidence. Cancer Treat Rev 72: 28-36, 2019.

8. McKeage MJ: Lobaplatin: A new antitumour platinum drug. Expert Opin Investig Drugs 10: 119-128, 2001.

9. Dawson LA and Lawrence TS: The role of radiotherapy in the treatment of liver metastases. Cancer J 10: 139-144, 2004.

10. Li C, Zhang F, Zhang W, Zhang L, Huang Z and Wu P: Feasibility of (125)I brachytherapy combined with sorafenib treatment in patients with multiple lung metastases after liver transplantation for hepatocellular carcinoma. J Cancer Res Clin Oncol 136 1633-1640, 2010

11. Zhang L, Mu W, Hu CF and Huang XQ: Treatment of portal vein tumor thrombus using ${ }^{125}$ Iodine seed implantation brachytherapy. World J Gastroenterol 16: 4876-4879, 2010.

12. Meng N, Jiang YL, Wang JJ, Ran WQ, Yuan HS, Qu A, Jiang P and Yang RJ: Permanent implantation of iodine-125 seeds as a salvage therapy for recurrent head and neck carcinoma after radiotherapy. Cancer Invest 30: 236-242, 2012.

13. Wang KX, Jin ZD, Du YQ, Zhan XB, Zou DW, Liu Y, Wang D, Chen J, Xu C and Li ZS: EUS-guided celiac ganglion irradiation with iodine-125 seeds for pain control in pancreatic carcinoma: A prospective pilot study. Gastrointest Endosc 76: 945-952, 2012

14. Litière S, Collette $\mathrm{S}$, de Vries EG, Seymour L and Bogaerts J: RECIST-learning from the past to build the future. Nat Rev Clin Oncol 14: 187-192, 2017.
15. Alterovitz R, Pouliot J, Taschereau R, Hsu IC and Goldberg K: Simulating needle insertion and radioactive seed implantation for prostate brachytherapy. Stud Health Technol Inform 94: 19-25, 2003.

16. Wang S, Shi G and Meng X: Clinical curative effect of percutaneous vertebroplasty combined with 125I-seed implantation in treating spinal metastatic tumor. Pak J Pharm Sci 28: S1039-S1042, 2015.

17. Lazarescu GR and Battista JJ: Analysis of the radiobiology of ytterbium-169 and iodine-125 permanent brachytherapy implants. Phys Med Biol 42: 1727-1736, 1997.

18. Zhuang HQ, Wang JJ, Liao AY, Wang JD and Zhao Y: The biological effect of 125I seed continuous low dose rate irradiation in CL187 cells. J Exp Clin Cancer Res 28: 12, 2009.

19. Nakamura H, Yasui Y, Saito N, Tachibana A, Komatsu K and Ishizaki K: DNA repair defect in AT cells and their hypersensitivity to low-dose-rate radiation. Radiat Res 165: 277-282, 2006.

20. Meigooni AS and Nath R: Response to Comment on 'Dosimetry of interstitial brachytherapy sources: Recommendation of the AAPM Radiation therapy committee task group 43' [Med. Phys. 26,2514 (1999)]. American association of physicists in medicine. Med Phys 27: 265, 2000.

21. Chen K, Xia Y, Wang H, Xiao F, Xiang G and Shen F: Adjuvant iodine-125 brachytherapy for hepatocellular carcinoma after complete hepatectomy: A randomized controlled trial. PLoS One 8: e57397, 2013.

22. Nag S, DeHaan M, Scruggs G, Mayr N and Martin EW: Long-term follow-up of patients of intrahepatic malignancies treated with iodine-125 brachytherapy. Int J Radiat Oncol Biol Phys 64: 736-744, 2006.

23. Martinez-Monge R, Nag S, Nieroda CA and Martin EW: Iodine-125 brachytherapy in the treatment of colorectal adenocarcinoma metastatic to the liver. Cancer 85: 1218-1225, 1999.

24. Zhang FJ, Li CX, Wu PH, Li K, Huang JH, Fan WJ, Zhang L, Gu YK, Lu MJ, Wu YX and Wang JJ: Radioactive seed 125I implantation in treating recurrence and metastasis after liver transplantation in hepatoma. Zhonghua Yi Xue Za Zhi 87: 956-959, 2007 (In Chinese).

25. Zhang FJ, Li CX, Zhang L, Wu PH, Jiao DC and Duan GF: Short-to mid-term evaluation of CT-guided 125I brachytherapy on intra-hepatic recurrent tumors and/or extra-hepatic metastases after liver transplantation for hepatocellular carcinoma. Cancer Biol Ther 8: 585-590, 2009.

26. Zhang FJ, Wu PH, Zhao M, Huang JH, Fan WJ, Gu YK, Liu J, Zhang L and Lu MJ: CT guided radioactive seed 125I implantation in treatment of pancreatic cancer. Zhonghua Yi Xue Za Zhi 86: 223-227, 2006 (In Chinese). 